УДК 514.822

\title{
Solving Yang-Mills Equations for 4-metrics of Petrov Types II, N, III
}

\author{
Leonid N. Krivonosov* \\ Nizhny Novgorod State Technical University \\ Minina, 24, Nizhny Novgorod, 603950 \\ Russia \\ Vyacheslav A. Lukyanov ${ }^{\dagger}$
}

Nizhny Novgorod State Technical University, Zavolzhsky branch, Pavlovskogo, 1a, Zavolzhye, Nizhegorodskaya region, 606520,

Russia

Received 10.06.2014, received in revised form 02.07.2014, accepted 30.08.2014

We have produced 4 series of 4-metrics satisfying Yang-Mills equations for each of types II, N, III.

Keywords: Einstein equations, Yang-Mills equations, manifold with conformal connection with torsion and without torsion.

\section{Introduction}

According to A. Z. Petrov's algebraic classification, Weyl tensor $C_{i m n}^{j}$ of conformal curvature of the square-law differential form of 4 variables is subdivided into three types I, II, III and three subtypes D, O, N (see [1,2]). In case of Einstein metrics its curvature tensor $R_{i m n}^{j}$ is subdivided into the same 6 kinds, while Weyl tensor and Riemann tensor are always of the same kind. Therefore in all cases it is possible to be limited to metric classification by the type of its Weyl tensor. It is clear, that this classification conformally invariant.

The subtype $\mathrm{O}$ means, that Weyl tensor vanishes, i.e. the metric is conformally flat. All conformally flat metrics automatically satisfy Yang-Mills equations. The equations for conformally flat metrics are much easier than Yang-Mills equations. Therefore this kind of metrics does not represent any interest from the point of view of searching solutions of Yang-Mills equations (though the most discussed in cosmology Robertson-Walker metric which is the solution of Friedmann equations belongs to type O). Solutions of Yang-Mills equations for kinds I, D and $\mathrm{O}$ already took place in our works. All central-symmetric metrics belong to kind $\mathrm{D}$ or $\mathrm{O}$. The full solution of Yang-Mills equations for central-symmetric metric has been found in [3]. In [4] solutions of Yang-Mills equations for the metric

$$
\psi=-d t^{2}+a^{2}(t) d x^{2}+b^{2}(t) d y^{2}+c^{2}(t) d z^{2}
$$

were searched. If $a \neq b \neq c$ this metric is referred to the types I or O, if $b=c-$ to the types D or O. In particular, when $a=t^{\alpha_{1}}, b=t^{\alpha_{2}}, c=t^{\alpha_{3}}$ the metric satisfies Yang-Mills equations, if

$$
\left(\alpha_{1}\right)^{2}+\left(\alpha_{2}\right)^{2}+\left(\alpha_{3}\right)^{2}-1=\frac{1}{2}\left(\alpha_{1}+\alpha_{2}+\alpha_{3}-1\right)^{2} .
$$

\footnotetext{
*oxyzt2@ya.ru

†oxyzt@ya.ru

(c) Siberian Federal University. All rights reserved
} 
When $\alpha_{1} \neq \alpha_{2} \neq \alpha_{3}$ we obtain a metric of type I, when $\alpha_{2}=\alpha_{3} \neq 0$, we get metric of subtype $\mathrm{D}$, when $\alpha_{1}=1, \alpha_{2}=\alpha_{3}=0$ - metric of subtype $\mathrm{O}$.

Therefore the purpose of present paper is searching the solutions of Yang-Mills equations for the remained three types II, N, III. In the modern literature such solutions have already been met. In particular, the solutions of Yang-Mills equations are found in [5] for homogeneous (i.e. allowing 3-parametrical invariancy group) Fefferman metric. The solution is

$$
g=d x^{2}+d y^{2}+\frac{2}{3}\left(y^{3} d u-d x\right)\left(y d r+\frac{1}{9} y^{3} d u+\frac{11}{9} d x\right) .
$$

Authors of that work do not notice that the metric is of the type N, since their paper has another purpose.

In this article we adhere to same tactics: Yang-Mills equations are made and solved only for the metrics allowing not less, than 2-parametrical invariancy group. Only in this case there is a hope to receive "solvable" Yang-Mills equations. It has appeared, that for metrics of type $\mathrm{N}$ the Yang-Mills equation most often are solvable, and with the big arbitrariness. For metrics of type III classes of metrics with solvable Yang-Mills equations are also rather easily found. The greatest difficulties for authors have caused searches of metrics of type II with solvable Yang-Mills equations since more often for such metrics the Yang-Mills equation do not allow to solve themselves though the solution may exist with arbitrariness in several functions. But if in addition to impose a stationary curvatures equality condition (A. Z. Petrov's terminology $[1$, Section 17]) the metric of a type II turns into the metrics of type N, or of type III, and the equations are often can be solved.

Further for brevity we will apply the term "Yang-Mills metric" to the metrics, satisfying Yang-Mills equations.

\section{Solving Yang-Mills equations for metrics of type $\mathrm{N}$}

1. We will begin with a metric of the type II

$$
\psi=2 d t(g(y) d x+h(t) d y+f(t, y, z) d t)+d y^{2}+d z^{2},
$$

to illustrate, how the structure of Yang-Mills equations improves when type II turns into type N. Put

$$
\begin{aligned}
\omega^{1} & =\left(\frac{1}{2}-f\right) d t-g d x-h d y, & \omega^{3}=d y, \\
\omega^{2} & =\left(\frac{1}{2}+f\right) d t+g d x+h d y, & \omega^{4}=d z .
\end{aligned}
$$

In this case $\psi=\eta_{i j} \omega^{i} \omega^{j}$, where $\eta_{i j}-$ Minkowski tensor with signature $(-+++)$. We compute components of conformal connection matrix

$$
\Omega=\left(\begin{array}{cccccc}
0 & \omega_{1} & \omega_{2} & \omega_{3} & \omega_{4} & 0 \\
\omega^{1} & 0 & \omega_{1}^{2} & \omega_{1}^{3} & \omega_{1}^{4} & \omega_{1} \\
\omega^{2} & \omega_{1}^{2} & 0 & -\omega_{2}^{3} & -\omega_{2}^{4} & -\omega_{2} \\
\omega^{3} & \omega_{1}^{3} & \omega_{2}^{3} & 0 & -\omega_{3}^{4} & -\omega_{3} \\
\omega^{4} & \omega_{1}^{4} & \omega_{2}^{4} & \omega_{3}^{4} & 0 & -\omega_{4} \\
0 & \omega^{1} & -\omega^{2} & -\omega^{3} & -\omega^{4} & 0
\end{array}\right)
$$


according to the standard scheme [3, p. 351-352]. We will denote partial derivatives $\frac{\partial f}{\partial t}=f_{t}$, $\frac{\partial f}{\partial y}=f_{y}$ etc. At first we find external differentials

$$
\begin{aligned}
& d \omega^{1}=-d \omega^{2}=f_{z}\left(\omega^{1}+\omega^{2}\right) \wedge \omega^{4}+\left[f_{y}-u\left(\frac{1}{2}+f\right)-h_{t}\right] \omega^{1} \wedge \omega^{3}+ \\
& +\left[f_{y}+u\left(\frac{1}{2}-f\right)-h_{t}\right] \omega^{2} \wedge \omega^{3}, \quad d \omega^{3}=d \omega^{4}=0, \quad u \stackrel{\text { def }}{=} \frac{g_{y}}{g}
\end{aligned}
$$

Then we calculate Pfaffian forms of Christoffel for the metrics (1)

$$
\begin{aligned}
& \omega_{1}^{2}=\frac{1}{2} u \omega^{3}, \quad \omega_{1}^{3}=\left(-f_{y}+u f+h_{t}\right)\left(\omega^{1}+\omega^{2}\right)+\frac{1}{2} u \omega^{1}, \quad \omega_{3}^{4}=0, \\
& \omega_{2}^{3}=\left(-f_{y}+u f+h_{t}\right)\left(\omega^{1}+\omega^{2}\right)-\frac{1}{2} u \omega^{2}, \quad \omega_{1}^{4}=\omega_{2}^{4}=-f_{z}\left(\omega^{1}+\omega^{2}\right) .
\end{aligned}
$$

Further we find external forms of Riemann curvature

$$
\begin{aligned}
& R_{1}^{2}=-\frac{1}{4} u^{2} \omega^{1} \wedge \omega^{2}, \quad R_{3}^{4}=0, \\
& R_{1}^{3}=\left(f_{y z}-\frac{1}{2} u f_{z}\right)\left(\omega^{1}+\omega^{2}\right) \wedge \omega^{4}+\left(f_{y}-u f\right)_{y}\left(\omega^{1}+\omega^{2}\right) \wedge \omega^{3}-\left(\frac{1}{2} u_{y}+\frac{1}{4} u^{2}\right) \omega^{1} \wedge \omega^{3}, \\
& R_{2}^{3}=\left(f_{y z}-\frac{1}{2} u f_{z}\right)\left(\omega^{1}+\omega^{2}\right) \wedge \omega^{4}+\left(f_{y}-u f\right)_{y}\left(\omega^{1}+\omega^{2}\right) \wedge \omega^{3}+\left(\frac{1}{2} u_{y}+\frac{1}{4} u^{2}\right) \omega^{2} \wedge \omega^{3}, \\
& R_{1}^{4}=R_{2}^{4}=f_{z z}\left(\omega^{1}+\omega^{2}\right) \wedge \omega^{4}+\left(f_{y z}-\frac{1}{2} u f_{z}\right)\left(\omega^{1}+\omega^{2}\right) \wedge \omega^{3} .
\end{aligned}
$$

We compute components of Ricci tensor $R_{i j}=R_{i j k}^{k}$ and its trace $R=\eta^{i j} R_{i j}$

$$
\begin{gathered}
R_{12}=\Delta f-(u f)_{y}, \quad R_{11}=R_{12}-\frac{1}{2}\left(u_{y}+u^{2}\right), \quad R_{44}=0, \\
R_{22}=R_{12}+\frac{1}{2}\left(u_{y}+u^{2}\right), \quad R_{33}=u_{y}+\frac{1}{2} u^{2}, \quad R=2 u_{y}+\frac{3}{2} u^{2} .
\end{gathered}
$$

Here $\Delta$ is Laplacian on variables $y$ and $z$. Hence, using formula $b_{i j}=\frac{1}{2} R_{i j}-\frac{1}{12} R \eta_{i j}$, we find (nonzero) components of Pfaffian forms $\omega_{i}=b_{i j} \omega^{j}$

As a result

$$
\begin{aligned}
b_{12}=\frac{1}{2}\left(\Delta f-(u f)_{y}\right), & b_{11}=b_{12}-K, \\
b_{22}=b_{12}+K, & b_{33}=\frac{1}{3} u_{y}+\frac{1}{8} u^{2}, \\
b_{44}=-\frac{1}{6} u_{y}-\frac{1}{8} u^{2}, & K=\frac{1}{12} u_{y}+\frac{1}{8} u^{2} .
\end{aligned}
$$

$$
\omega_{1}=b_{12}\left(\omega^{1}+\omega^{2}\right)-K \omega^{1}, \omega_{2}=b_{12}\left(\omega^{1}+\omega^{2}\right)+K \omega^{2}, \omega_{3}=b_{33} \omega^{3}, \omega_{4}=b_{44} \omega^{4},
$$

and the matrix of conformal connection is completely defined. Now we compute components of a matrix of conformal curvature

$$
\Phi=\left(\begin{array}{cccccc}
0 & \Phi_{1} & \Phi_{2} & \Phi_{3} & \Phi_{4} & 0 \\
0 & 0 & \Phi_{1}^{2} & \Phi_{1}^{3} & \Phi_{1}^{4} & \Phi_{1} \\
0 & \Phi_{1}^{2} & 0 & -\Phi_{2}^{3} & -\Phi_{2}^{4} & -\Phi_{2} \\
0 & \Phi_{1}^{3} & \Phi_{2}^{3} & 0 & -\Phi_{3}^{4} & -\Phi_{3} \\
0 & \Phi_{1}^{4} & \Phi_{2}^{4} & \Phi_{3}^{4} & 0 & -\Phi_{4} \\
0 & 0 & 0 & 0 & 0 & 0
\end{array}\right)
$$


in correspondence with formulas $\Phi_{i}^{j}=R_{i}^{j}+\omega^{j} \wedge \omega_{i}+\eta^{j k} \omega_{k} \wedge \eta_{i s} \omega^{s}$ and $\Phi_{i}=d \omega_{i}+\omega_{k} \wedge \omega_{i}^{k}$

$$
\begin{aligned}
& \Phi_{1}^{2}=R_{1}^{2}+\omega^{2} \wedge \omega_{1}-\omega_{2} \wedge \omega^{1}=2 S \omega^{1} \wedge \omega^{2}, \quad \Phi_{3}^{4}=-2 S \omega^{3} \wedge \omega^{4}, \\
& \Phi_{1}^{3}=T\left(\omega^{1}+\omega^{2}\right) \wedge \omega^{3}-S \omega^{1} \wedge \omega^{3}+P\left(\omega^{1}+\omega^{2}\right) \wedge \omega^{4}, \\
& \Phi_{1}^{4}=P\left(\omega^{1}+\omega^{2}\right) \wedge \omega^{3}-T\left(\omega^{1}+\omega^{2}\right) \wedge \omega^{4}-S \omega^{1} \wedge \omega^{4}, \\
& \Phi_{2}^{3}=T\left(\omega^{1}+\omega^{2}\right) \wedge \omega^{3}+S \omega^{2} \wedge \omega^{3}+P\left(\omega^{1}+\omega^{2}\right) \wedge \omega^{4}, \\
& \Phi_{2}^{4}=P\left(\omega^{1}+\omega^{2}\right) \wedge \omega^{3}-T\left(\omega^{1}+\omega^{2}\right) \wedge \omega^{4}+S \omega^{2} \wedge \omega^{4},
\end{aligned}
$$

where we denote $S \stackrel{\text { def }}{=} \frac{1}{12} u_{y}, T \stackrel{\text { def }}{=} \frac{1}{2}\left(f_{y y}-f_{z z}\right)-\frac{1}{2}(u f)_{y}, P \stackrel{\text { def }}{=} f_{y z}-\frac{1}{2} u f_{z}$. It means, that components of Weyl tensor $C_{i m n}^{j}=\Phi_{i m n}^{j}$ are equal to $\Phi_{112}^{2}=2 S, \Phi_{113}^{3}=T-S, \Phi_{123}^{3}=T$ etc.

$$
\begin{aligned}
& \Phi_{1}=X\left(\omega^{1}+\omega^{2}\right) \wedge \omega^{3}+Z \omega^{1} \wedge \omega^{3}+Y\left(\omega^{1}+\omega^{2}\right) \wedge \omega^{4}, \quad \Phi_{3}=0, \\
& \Phi_{2}=X\left(\omega^{1}+\omega^{2}\right) \wedge \omega^{3}-Z \omega^{2} \wedge \omega^{3}+Y\left(\omega^{1}+\omega^{2}\right) \wedge \omega^{4}, \quad \Phi_{4}=\left(b_{44}\right)_{y} \omega^{3} \wedge \omega^{4},
\end{aligned}
$$

where for brevity we denote $X \stackrel{\text { def }}{=}-\left(b_{12}\right)_{y}+\frac{1}{2} b_{12} u+\frac{1}{4} u_{y}\left(f_{y}-u f\right), Y \stackrel{\text { def }}{=}-\left(b_{12}\right)_{z}-$ $\frac{1}{4} f_{z}\left(u_{y}+u^{2}\right), Z \stackrel{\text { def }}{=} \frac{1}{12} u_{y y}+\frac{1}{8} u_{y} u$. Petrov matrix $Q(\lambda)[1$, formula (18.14)], made with the help of the components of Weyl tensor, looks like

$$
Q(\lambda)=\left(\begin{array}{ccc}
-2 S+\lambda & 0 & 0 \\
0 & -T+S+i P+\lambda & -P-i T \\
0 & -P-i T & T+S-i P+\lambda
\end{array}\right) .
$$

It is a matrix of type II with 1 -fold eigenvalue $\lambda_{1}=2 S$ and double eigenvalue $\lambda_{2}=-S$. But if $S=0$ it is of type N.

To compose Yang-Mills equations we will write components of a dual matrix $* \Phi(*$ is Hodge star operator) [3, p. 352, item 6$]$

$$
\begin{aligned}
& * \Phi_{1}^{2}=2 S \omega^{3} \wedge \omega^{4}, * \Phi_{3}^{4}=2 S \omega^{1} \wedge \omega^{2}, * \Phi_{4}=-\left(b_{44}\right)_{y} \omega^{1} \wedge \omega^{2}, \\
& * \Phi_{1}^{3}=P\left(\omega^{1}+\omega^{2}\right) \wedge \omega^{3}-T\left(\omega^{1}+\omega^{2}\right) \wedge \omega^{4}+S \omega^{2} \wedge \omega^{4}, \\
& * \Phi_{1}^{4}=-T\left(\omega^{1}+\omega^{2}\right) \wedge \omega^{3}-S \omega^{2} \wedge \omega^{3}-P\left(\omega^{1}+\omega^{2}\right) \wedge \omega^{4}, \\
& * \Phi_{2}^{3}=-T\left(\omega^{1}+\omega^{2}\right) \wedge \omega^{4}-S \omega^{1} \wedge \omega^{4}+P\left(\omega^{1}+\omega^{2}\right) \wedge \omega^{3}, \\
& * \Phi_{2}^{4}=-P\left(\omega^{1}+\omega^{2}\right) \wedge \omega^{4}-T\left(\omega^{1}+\omega^{2}\right) \wedge \omega^{3}+S \omega^{1} \wedge \omega^{3}, \\
& * \Phi_{1}=-X\left(\omega^{1}+\omega^{2}\right) \wedge \omega^{4}-Z \omega^{2} \wedge \omega^{4}+Y\left(\omega^{1}+\omega^{2}\right) \wedge \omega^{3}, \\
& * \Phi_{2}=-X\left(\omega^{1}+\omega^{2}\right) \wedge \omega^{4}+Z \omega^{1} \wedge \omega^{4}+Y\left(\omega^{1}+\omega^{2}\right) \wedge \omega^{3}, * \Phi_{3}=0 .
\end{aligned}
$$

Yang-Mills equations $d * \Phi+\Omega \wedge * \Phi-* \Phi \wedge \Omega=0$ for external forms $* \Phi_{3}$ and $* \Phi_{4}$ are

$$
d * \Phi_{3}+\omega_{k} \wedge * \Phi_{3}^{k}-* \Phi_{k} \wedge \omega_{3}^{k}=0, \quad d * \Phi_{4}+\omega_{k} \wedge * \Phi_{4}^{k}-* \Phi_{k} \wedge \omega_{4}^{k}=0 .
$$

In components they give two equations

$$
2 S\left(b_{44}-K\right)+Z u=0, \quad 2 S\left(K-b_{33}\right)-\left(b_{44}\right)_{y} u-\left(b_{44}\right)_{y y}=0 .
$$

These equations are expressed only through the function $u=\frac{g_{y}}{g}$ :

$$
\begin{aligned}
u_{y y} u-\frac{1}{2}\left(u_{y}\right)^{2}+u^{2} u_{y} & =0, \\
u_{y y y}+\frac{5}{2} u_{y y} u+\frac{5}{4}\left(u_{y}\right)^{2}+\frac{3}{2} u^{2} u_{y} & =0 .
\end{aligned}
$$


If to denote $L$ and $Q$ the left parts of these equations, the formula $Q=\frac{1}{u} L_{u}+\frac{3}{2} L$ shows that the second of these equations is a differential consequence of the first.

Yang-Mills equation for the external form $* \Phi_{1}$ produces

$$
\begin{gathered}
X_{y}+Z\left(f_{y}-u f-h_{t}\right)+Y_{z}+2 b_{12} S+\left(b_{33}-b_{44}\right) T-\frac{1}{2} u X=0 \\
X_{y}+Z_{y}+Z\left(f_{y}+\left(\frac{1}{2}-f\right) u-h_{t}\right)+Y_{z}+2 b_{12} S+ \\
+2 S K-\left(b_{33}+b_{44}\right) S+\left(b_{33}-b_{44}\right) T-\frac{1}{2} u X=0 .
\end{gathered}
$$

Their difference on account of (7) vanishes, therefore it is possible to leave only the first equation. In detail it looks like

$$
\begin{gathered}
\frac{\Delta \Delta f}{-2}+u \Delta f_{y}+\left(\frac{u_{y}}{3}-\frac{u^{2}}{2}\right) \Delta f+2 u_{y} f_{y y}+\frac{11}{6}\left(u_{y y}-u_{y} u\right) f_{y}+ \\
+\left(\frac{u_{y y y}}{2}-\frac{5\left(u_{y}\right)^{2}}{6}-\frac{5 u_{y y} u}{6}\right) f-h_{t}\left(\frac{u_{y y}}{12}+\frac{u_{y} u}{8}\right)=0 .
\end{gathered}
$$

The Yang-Mills equation for the external form $* \Phi_{2}$ does not result new equations. Thus, the whole system of Yang-Mills equations is reduced to (7) and (8). The equation (8) serves to find function $f$. Though it is linear it's difficult to specify its solution without additional restrictions. However if the type of the metric (1) turns from II into $\mathrm{N}$, i.e. at $S=0$, that is equivalent to $u_{y}=0, u=\alpha, g=\beta e^{\alpha y}$, where $\alpha, \beta=$ const, then equation (7) is satisfied identically, and equation (8) becomes good enough

$$
\Delta \Delta f-2 \alpha \Delta f_{y}+\alpha^{2} \Delta f=0
$$

In particular, at $\alpha=0$ it turn into the well known equation $\Delta \Delta f=0$. It is easy to specify its solutions in the polynomial form.

Another special case, if $f$ does not depend on $y$. Then equation (9) leads to $\frac{\partial^{4} f}{\partial z^{4}}+\alpha^{2} \frac{\partial^{2} f}{\partial z^{2}}=0$. Its general solution is $f=\lambda \cos \alpha z+\mu \sin \alpha z+\delta z+\varepsilon$, where $\lambda, \mu, \delta, \varepsilon$ are arbitrary functions of $t$.

In the case, when $f$ does not depend on $z$, equation (9) is reduced to $\frac{\partial^{4} f}{\partial y^{4}}-2 \alpha \frac{\partial^{3} f}{\partial y^{3}}+\alpha^{2} \frac{\partial^{2} f}{\partial y^{2}}=$ 0, i.e. $f=(\lambda y+\mu) e^{\alpha y}+\delta y+\varepsilon$, where $\lambda, \mu, \delta, \varepsilon$ are arbitrary functions of $t$.

Solutions of equations (7) and (8), not leading to type N, will be examined in the following section.

Notice, that metric (1) will be Einstein metric, i.e. $R_{i j}=\varkappa \eta_{i j}$, iff $g=$ const $\neq 0, \Delta f=0$. It follows from (4).

Further we will omit detailed computations and will write only matrixes of conformal connection $\Omega$ and curvature $\Phi$ and final equations.

2. Let's investigate metric

$$
\psi=2 d t(d x+B(t, y) d t)+(A(t, y) d y)^{2}+d z^{2}
$$

We denote with dot differentiation with respect to $t$, and the stroke' denotes a derivative with 
respect to $y$. The matrix of conformal connection (2) is

$$
\begin{aligned}
& \omega^{1}=\left(\frac{1}{2}-B\right) d t-d x, \quad \omega^{2}=\left(\frac{1}{2}+B\right) d t+d x, \quad \omega^{3}=A d y, \quad \omega^{4}=d z \\
& \omega_{1}^{2}=\omega_{1}^{4}=\omega_{2}^{4}=\omega_{3}^{4}=0, \quad \omega_{1}^{3}=\omega_{2}^{3}=-\frac{B^{\prime}}{A}\left(\omega^{1}+\omega^{2}\right)+\frac{\dot{A}}{A} \omega^{3}, \\
& \omega_{1}=\omega_{2}=\frac{1}{2} K\left(\omega^{1}+\omega^{2}\right), \quad \omega_{3}=\omega_{4}=0,
\end{aligned}
$$

where $K \stackrel{\text { def }}{=} \frac{1}{A}\left(\frac{B^{\prime}}{A}\right)^{\prime}+\frac{\ddot{A}}{A}$. The matrix of conformal curvature (5) has components

$$
\begin{aligned}
& \Phi_{1}^{2}=\Phi_{3}^{4}=0, \quad \Phi_{1}^{3}=\Phi_{2}^{3}=\frac{1}{2} K\left(\omega^{1}+\omega^{2}\right) \wedge \omega^{3}, \quad \Phi_{3}=\Phi_{4}=0, \\
& \Phi_{1}^{4}=\Phi_{2}^{4}=-\frac{1}{2} K\left(\omega^{1}+\omega^{2}\right) \wedge \omega^{4}, \quad \Phi_{1}=\Phi_{2}=-\frac{K^{\prime}}{2 A}\left(\omega^{1}+\omega^{2}\right) \wedge \omega^{3} .
\end{aligned}
$$

Petrov matrix looks like

It is a matrix of type $\mathrm{N}$.

$$
Q(\lambda)=\left(\begin{array}{ccc}
\lambda & 0 & 0 \\
0 & -\frac{1}{2} K+\lambda & -\frac{i}{2} K \\
0 & -\frac{i}{2} K & \frac{1}{2} K+\lambda
\end{array}\right)
$$

Yang-Mills equation (6) for forms $* \Phi_{3}$ and $* \Phi_{4}$ are satisfied identically, and equations for forms $* \Phi_{1}$ and $* \Phi_{2}$ result in the same equation $\left(\frac{K^{\prime}}{A}\right)^{\prime}=0$, or in the unwrapped shape $\left[\frac{1}{A}\left(\frac{1}{A}\left(\frac{B^{\prime}}{A}\right)^{\prime}+\frac{\ddot{A}}{A}\right)^{\prime}\right]^{\prime}=0$. We have derived one equation on two functions $A$ and $B$ of two variables. An arbitrariness of solutions is great: one of functions $A$ or $B$ can be unrestricted. It is easy to specify many particular solutions in an explicit form. For example, if $A$ does not depend on $y$, then $B=\alpha y^{3}+\beta y^{2}+\gamma y+\delta$, where $\alpha, \beta, \gamma, \delta$ are arbitrary functions of $t$.

3. For metric

$$
\psi=2 d t(d x-\varepsilon z d y)+(a(t) d y+b(t) d z)^{2}+(c(t) d z)^{2},
$$

where $\varepsilon=$ const, the matrix of conformal connection (2) has components

$$
\begin{aligned}
& \omega^{1}=\frac{1}{2} d t-d x+\varepsilon z d y, \omega^{2}=\frac{1}{2} d t+d x-\varepsilon z d y, \omega^{3}=a d y+b d z, \omega^{4}=c d z \\
& \omega_{1}^{2}=0, \omega_{1}^{3}=\omega_{2}^{3}=\frac{\dot{a}}{a} \omega^{3}+\frac{1}{2}\left(P-\frac{\varepsilon}{a c}\right) \omega^{4}, \omega_{3}^{4}=\frac{1}{2}\left(P+\frac{\varepsilon}{a c}\right)\left(\omega^{1}+\omega^{2}\right) \\
& \omega_{1}^{4}=\omega_{2}^{4}=\frac{1}{2}\left(P+\frac{\varepsilon}{a c}\right) \omega^{3}+\frac{\dot{c}}{c} \omega^{4}, \omega_{1}=\omega_{2}=K\left(\omega^{1}+\omega^{2}\right), \omega_{3}=\omega_{4}=0
\end{aligned}
$$

where $P \stackrel{\text { def }}{=} \frac{\dot{b}}{c}-\frac{\dot{a} b}{a c}, K \stackrel{\text { def }}{=} \frac{1}{2}\left(\frac{\ddot{a}}{a}+\frac{\ddot{c}}{c}+\frac{1}{2} P^{2}-\frac{1}{2} \frac{\varepsilon}{a^{2} c^{2}}\right)$.

Components of conformal curvature matrix (5) are

$$
\begin{aligned}
& \Phi_{1}^{2}=\Phi_{3}^{4}=\Phi_{1}=\Phi_{2}=\Phi_{3}=\Phi_{4}=0, \\
& \Phi_{1}^{3}=\Phi_{2}^{3}=S\left(\omega^{1}+\omega^{2}\right) \wedge \omega^{3}+T\left(\omega^{1}+\omega^{2}\right) \wedge \omega^{4}, \\
& \Phi_{1}^{4}=\Phi_{2}^{4}=T\left(\omega^{1}+\omega^{2}\right) \wedge \omega^{3}-S\left(\omega^{1}+\omega^{2}\right) \wedge \omega^{4},
\end{aligned}
$$


where $S \stackrel{\text { def }}{=} \frac{1}{2}\left(\frac{\ddot{a}}{a}-\frac{\ddot{c}}{c}-P^{2}-P \frac{\varepsilon}{a c}\right), T \stackrel{\text { def }}{=} \frac{1}{2} \dot{P}+\frac{\ddot{a}}{a} P+\frac{\varepsilon \dot{a}}{2 a^{2} c}-\frac{\varepsilon \dot{c}}{a c^{2}}$.

Yang-Mills equations for the metric (11) are satisfied identically. The metric has a type N. It is Einsteinian, if $K=0$.

Many known metrics for which Einstein equations were solved, are of type $\mathrm{N}$ and are reduced to metrics (1), (10) or (11). We will bring several examples.

4. Peres metric

$$
\psi=-d t^{2}+d x^{2}+d y^{2}+d z^{2}+f(t-x, y, z)(d t-d x)^{2}
$$

after the substitution of variables $t-x=-u, t+x=2 v, F(u, y, z)=\frac{1}{2} f(t-x, y, z)$ turns into

$$
\psi=2 d u(d v+F(u, y, z) d u)+d y^{2}+d z^{2}
$$

which is a special case of metric (1) with $g(y)=1$ and $h(u)=0$. Therefore for Peres metric Yang-Mills equation consist of one equation (9) $\Delta \Delta F=0$, where $\Delta$ is Laplacian with respect to variables $y$ and $z$.

5. Takeno metric

$$
\psi=-(P+S) d t^{2}+2 S d t d x+(P-S) d x^{2}+A d y^{2}+2 B d y d z+C d z^{2},
$$

where $A, B, C, P, S$ are functions of $t-x$, after the substitution of variables $u=t-x, v=t+x$ turns into

$$
\psi=-P(u) d u d v-S(u) d u^{2}+A(u) d y^{2}+2 B(u) d y d z+C(u) d z^{2}
$$

Now, instead of $v$, we introduce a new variable $w=-\frac{1}{2}\left(v+\int \frac{S(u)}{P(u)} d u\right)$, then we introduce a new parameter $\tau=\int P(u) d u$. As a result,

$$
\psi=2 d \tau d w+A(u(\tau)) d y^{2}+2 B(u(\tau)) d y d z+C(u(\tau)) d z^{2}
$$

i.e. special case of the metric (11). Yang-Mills equations and Petrov type of the metric are invariant with respect to performed operations, that's why the Takeno metric is of type N and identically satisfies Yang-Mills equations.

6. Rosen metric

$$
\psi=-\exp (2 \mu)\left(d t^{2}-d x^{2}\right)+u^{2} \exp (2 \nu) d y^{2}+\exp (-2 \nu) d z^{2},
$$

where $\mu$ and $\nu$ are functions of $u=t-x$, is a special case of the metric

$$
\psi_{1}=D(u)\left(d t^{2}-d x^{2}\right)+A(u) d y^{2}+2 B(u) d y d z+C(u) d z^{2},
$$

and the latter is conformally equivalent to the metric (11) at $\varepsilon=0$. Therefore, Yang-Mills equations for the Rosen metric are satisfied identically.

7. Bondi-Piranha-Robinson metric

$$
\psi=d t^{2}-d x^{2}+\alpha d y^{2}+2 \beta d y d z+\gamma d z^{2},
$$

where $\alpha, \beta$ and $\gamma$ are functions of $t+x$, is obviously isomorphic to the metric (11) at $\varepsilon=0$. Therefore, Yang-Mills equations for this metric are satisfied identically. 


\section{Solving Yang-Mills equations for metrics of type II}

1. Let's construct and solve Yang-Mills equations for the metric

$$
\psi=2 d t(d x+A(t, z) d t)+\left(e^{\alpha t} C(z) d y\right)^{2}+d z^{2}, \quad \alpha=\text { const. }
$$

As we shall see, this metric can be of all types, except type I, and for types II, III, N YangMills equations admit explicit nontrivial solutions. Derivative with respect to $z$ is denoted by a stroke'.

Then we compute the components of the conformal connection matrix (2)

$$
\begin{aligned}
& \omega^{1}=\left(\frac{1}{2}-A\right) d t-d x, \omega^{2}=\left(\frac{1}{2}+A\right) d t+d x, \omega^{3}=C e^{\alpha t} d y, \omega^{4}=d z, \\
& \omega_{1}^{2}=0, \omega_{3}^{4}=-\frac{C^{\prime}}{C} \omega^{3}, \omega_{1}^{3}=\omega_{2}^{3}=\alpha \omega^{3}, \omega_{1}^{4}=\omega_{2}^{4}=-A^{\prime}\left(\omega^{1}+\omega^{2}\right) .
\end{aligned}
$$

The components of the Ricci tensor and its trace are

$$
\begin{gathered}
R_{11}=R_{22}=R_{12}=A^{\prime \prime}+\frac{C^{\prime}}{C} A^{\prime}+\alpha^{2}, \\
R_{14}=R_{24}=\alpha \frac{C^{\prime}}{C}, \quad R_{33}=R_{44}=\frac{C^{\prime \prime}}{C}, \quad R=2 \frac{C^{\prime \prime}}{C}
\end{gathered}
$$

The remaining 4 Pfaffian forms of the conformal connection matrix $\Omega$ are

$$
\begin{aligned}
& \omega_{1}=b_{12}\left(\omega^{1}+\omega^{2}\right)+\frac{C^{\prime \prime}}{6 C} \omega^{1}+\frac{1}{2} \alpha \frac{C^{\prime}}{C} \omega^{4}, \omega_{3}=\frac{C^{\prime \prime}}{3 C} \omega^{3}, \\
& \omega_{2}=b_{12}\left(\omega^{1}+\omega^{2}\right)-\frac{C^{\prime \prime}}{6 C} \omega^{2}+\frac{1}{2} \alpha \frac{C^{\prime}}{C} \omega^{4}, \\
& \omega_{4}=\alpha \frac{C^{\prime}}{2 C}\left(\omega^{1}+\omega^{2}\right)+\frac{C^{\prime \prime}}{3 C} \omega^{4}, b_{12}=\frac{1}{2}\left(A^{\prime \prime}+A^{\prime} \frac{C^{\prime}}{C}+\alpha^{2}\right) .
\end{aligned}
$$

Now we write down the components of the conformal curvature matrix (5), where for brevity $P \stackrel{\text { def }}{=}\left(A^{\prime \prime}-A^{\prime} \frac{C^{\prime}}{C}-\alpha^{2}\right)$

$$
\begin{aligned}
& \Phi_{1}^{2}=-\frac{C^{\prime \prime}}{3 C} \omega^{1} \wedge \omega^{2}+\alpha \frac{C^{\prime}}{2 C}\left(\omega^{1}+\omega^{2}\right) \wedge \omega^{4} \\
& \Phi_{1}^{3}=\left(\frac{C^{\prime \prime}}{6 C}-P\right) \omega^{1} \wedge \omega^{3}-P \omega^{2} \wedge \omega^{3}-\alpha \frac{C^{\prime}}{2 C} \omega^{3} \wedge \omega^{4}, \\
& \Phi_{2}^{3}=-P \omega^{1} \wedge \omega^{3}-\left(\frac{C^{\prime \prime}}{6 C}+P\right) \omega^{2} \wedge \omega^{3}-\alpha \frac{C^{\prime}}{2 C} \omega^{3} \wedge \omega^{4}, \\
& \Phi_{1}^{4}= P \omega^{2} \wedge \omega^{4}+\left(\frac{C^{\prime \prime}}{6 C}+P\right) \omega^{1} \wedge \omega^{4}+\alpha \frac{C^{\prime}}{2 C} \omega^{1} \wedge \omega^{2}, \\
& \Phi_{2}^{4}=\left(P-\frac{C^{\prime \prime}}{6 C}\right) \omega^{2} \wedge \omega^{4}+P \omega^{1} \wedge \omega^{4}+\alpha \frac{C^{\prime}}{2 C} \omega^{1} \wedge \omega^{2}, \\
& \Phi_{3}^{4}=\frac{C^{\prime \prime}}{3 C} \omega^{3} \wedge \omega^{4}-\alpha \frac{C^{\prime}}{2 C}\left(\omega^{1}+\omega^{2}\right) \wedge \omega^{3}, \\
& \Phi_{1}=-\left(\frac{C^{\prime \prime}}{6 C}\right)^{\prime} \omega^{1} \wedge \omega^{4}+\left(-b_{12}^{\prime}+\frac{A^{\prime} C^{\prime \prime}}{2 C}\right)\left(\omega^{1}+\omega^{2}\right) \wedge \omega^{4},
\end{aligned}
$$




$$
\begin{aligned}
& \Phi_{2}=\left(\frac{C^{\prime \prime}}{6 C}\right)^{\prime} \omega^{2} \wedge \omega^{4}+\left(-b_{12}^{\prime}+\frac{A^{\prime} C^{\prime \prime}}{2 C}\right)\left(\omega^{1}+\omega^{2}\right) \wedge \omega^{4}, \\
& \Phi_{3}=-\left(\frac{C^{\prime \prime}}{3 C}\right)^{\prime} \omega^{3} \wedge \omega^{4}+\frac{\alpha}{2}\left(\frac{C^{\prime}}{C}\right)^{\prime}\left(\omega^{1}+\omega^{2}\right) \wedge \omega^{3}, \\
& \Phi_{4}=-\frac{\alpha}{2}\left(\frac{C^{\prime}}{C}\right)^{\prime}\left(\omega^{1}+\omega^{2}\right) \wedge \omega^{4} .
\end{aligned}
$$

Petrov matrix $Q(\lambda)$ is

$$
\left(\begin{array}{ccc}
\frac{C^{\prime \prime}}{3 C}+\lambda & \frac{i \alpha C^{\prime}}{2 C} & -\frac{\alpha C^{\prime}}{2 C} \\
\frac{i \alpha C^{\prime}}{2 C} & -\frac{C^{\prime \prime}}{6 C}+P+\lambda & i P \\
-\frac{\alpha C^{\prime}}{2 C} & i P & -\frac{C^{\prime \prime}}{6 C}-P+\lambda
\end{array}\right)
$$

It has type II when $C^{\prime \prime} \neq 0, \frac{P C^{\prime \prime}}{C}+\frac{1}{2}\left(\frac{\alpha C^{\prime}}{C}\right)^{2} \neq 0 ;$ type D if $C^{\prime \prime} \neq 0, \frac{P C^{\prime \prime}}{C}+\frac{1}{2}\left(\frac{\alpha C^{\prime}}{C}\right)^{2}=0$; type III if $C^{\prime \prime}=0, \alpha C^{\prime} \neq 0$; type $\mathrm{N}$ if $C^{\prime \prime}=0, \alpha C^{\prime}=0, P \neq 0$; type $\mathrm{O}$ when $C^{\prime \prime}=0, \alpha C^{\prime}=0$, $P=0$.

Yang-Mills equation for the external form $* \Phi_{3}$ gives

$$
\left(\frac{C^{\prime \prime}}{C}\right)^{\prime \prime}-\frac{1}{2}\left(\frac{C^{\prime \prime}}{C}\right)^{2}=0
$$

Yang-Mills equation for the external form $* \Phi_{4}$ results

$$
\begin{aligned}
\left(\frac{C^{\prime \prime}}{C}\right)^{\prime} \frac{C^{\prime}}{C}-\frac{1}{2}\left(\frac{C^{\prime \prime}}{C}\right)^{2} & =0, \\
\alpha\left(\left(\frac{C^{\prime \prime}}{C}\right)^{\prime}-\frac{2}{3}\left(\frac{C^{\prime \prime} C^{\prime}}{C^{2}}\right)\right) & =0 .
\end{aligned}
$$

The remaining two equations for forms $* \Phi_{1}$ and $* \Phi_{2}$ with the help of the equalities (14)-(16) lead to

$$
\begin{gathered}
-\frac{1}{2} A^{(4)}-\frac{C^{\prime}}{C} A^{\prime \prime \prime}+\left(\frac{1}{2}\left(\frac{C^{\prime}}{C}\right)^{2}-\frac{2 C^{\prime \prime}}{3 C}\right) A^{\prime \prime}+ \\
+\left(-\frac{C^{\prime \prime \prime}}{6 C}+\frac{C^{\prime \prime} C^{\prime}}{C^{2}}-\frac{1}{2}\left(\frac{C^{\prime}}{C}\right)^{3}\right) A^{\prime}+\alpha^{2}\left(\left(\frac{C^{\prime}}{C}\right)^{2}-\frac{2 C^{\prime \prime}}{3 C}\right)=0 .
\end{gathered}
$$

Thus, the system of Yang-Mills equations is reduced to (14)-(17). To solve it, we first note that the equation (14) is a differential consequence of (15). Equation (15) allows reduction of order. It is equivalent to

$$
C^{\prime}=\left(\beta C^{2}+\gamma\right)^{\frac{2}{3}}
$$

where $\beta$ and $\gamma$ are constants.

Put $\alpha \neq 0$. Then, eliminating the third derivative from (15) and (16), we obtain

$$
\frac{C^{\prime \prime}}{C}\left(\frac{C^{\prime \prime}}{C}-\frac{4}{3}\left(\frac{C^{\prime}}{C}\right)^{2}\right)=0 .
$$


In equation (19) we initially set to zero the second factor. The resulting equation can be easily integrated

$$
C=\frac{1}{(\lambda z+\mu)^{3}}, \quad \lambda, \mu=\text { const. }
$$

This corresponds to (18) at $\gamma=0$. Substituting (20) to (17), we obtain the differential equation of Euler type

$$
-\frac{1}{2} A^{(4)}+\frac{3 \lambda}{\lambda z+\mu} A^{\prime \prime \prime}-\frac{7 \lambda^{2}}{2(\lambda z+\mu)^{2}} A^{\prime \prime}-\frac{25 \lambda^{3}}{2(\lambda z+\mu)^{3}} A^{\prime}+\frac{\alpha^{2} \lambda^{2}}{(\lambda z+\mu)^{2}}=0 .
$$

Its general solution

$$
A=\varepsilon_{1}+\varepsilon_{2} \ln \left|z+\frac{\mu}{\lambda}\right|+\left(\varepsilon_{3}+\varepsilon_{4} \ln \left|z+\frac{\mu}{\lambda}\right|\right)\left(z+\frac{\mu}{\lambda}\right)^{6}+\frac{\alpha^{2}}{32}\left(z+\frac{\mu}{\lambda}\right)^{2},
$$

where $\varepsilon_{1}, \varepsilon_{2}, \varepsilon_{3}, \varepsilon_{4}$ are arbitrary functions of $t$. By making the change of variable $z+\frac{\mu}{\lambda} \rightarrow z$, we obtain the final solution

$$
A=\varepsilon_{1}+\varepsilon_{2} \ln |z|+\left(\varepsilon_{3}+\varepsilon_{4} \ln |z|\right) z^{6}+\frac{\alpha^{2}}{32} z^{2}, \quad C=\frac{1}{(\lambda z)^{3}},
$$

depending on four arbitrary functions of the variable $t$. Since $C^{\prime \prime} \neq 0$ and $P \frac{C^{\prime \prime}}{C}+\frac{1}{2} \alpha\left(\frac{C^{\prime}}{C}\right)^{2} \neq 0$, this solution gives the metric of type II.

Although the solution (22) is obtained for $\alpha \neq 0$, it is also a solution in the case $\alpha=0$, but it is not a general solution, because equation (18) besides the solution (20) at $\gamma=0$, has other non-elementary solutions at $\gamma \neq 0$. But in the latter case we cannot find explicit solutions of the equation (17). Solution (22) in the case of $\alpha=0$ gives the metric of type II.

Now we consider the second possibility of the equality (19), $C^{\prime \prime}=0$, which is equivalent to $C=\lambda z+\mu$, where $\lambda, \mu=$ const. Substituting this in (17), we again obtain the equation of Euler type

$$
-\frac{1}{2} A^{(4)}-\frac{\lambda}{\lambda z+\mu} A^{\prime \prime \prime}+\frac{\lambda^{2}}{2(\lambda z+\mu)^{2}} A^{\prime \prime}-\frac{\lambda^{3}}{2(\lambda z+\mu)^{3}} A^{\prime}+\frac{\alpha^{2} \lambda^{2}}{(\lambda z+\mu)^{2}}=0 .
$$

In its general solution, we replace $z+\frac{\mu}{\lambda}$ with $z$ and get

$$
A=\varepsilon_{1}+\varepsilon_{2} \ln |z|+\left(\varepsilon_{3}+\varepsilon_{4} \ln |z|+\frac{\alpha^{2}}{4} \ln ^{2}|z|\right) z^{2}, \quad C=\lambda z .
$$

At $\alpha \neq 0$ this solution gives the metric of type III, and at $\alpha=0$ the metric of type $\mathrm{N}$, different from the metric of type $\mathrm{N}$ in section 1.

If $C=$ const equation (17) has a general solution $A=\varepsilon_{1}(t)+\varepsilon_{2}(t) z+\varepsilon_{3}(t) z^{2}+\varepsilon_{4}(t) z^{3}$. This solution gives the metric of type $\mathrm{N}$, but it is conformally equivalent to the special case of the metric (10).

From (13), it follows that (12) is Einstein metric in cases

$$
\begin{aligned}
& \text { 1) } \alpha \neq 0, C=\text { const, } A=-\frac{1}{2} \alpha^{2} z^{2}+\varepsilon_{1}(t) z+\varepsilon_{2}(t) ; \\
& 2) \alpha=0, C=\lambda z+\mu, A=\varepsilon_{1}(t) \ln \left|z+\frac{\mu}{\lambda}\right|+\varepsilon_{2}(t) .
\end{aligned}
$$

In both cases, the metric has a type N. 
2. Let's return to the metric (1) and Yang-Mills equations (7) and (8). We will show that under the additional condition: $f(t, y, z)$ doesn't depend on $z$, equation (8) already has explicit solutions. In this case it turns into

$$
\begin{aligned}
& -\frac{1}{2} f_{y y y y}+u f_{y y y}+\left(\frac{7 u_{y}}{3}-\frac{u^{2}}{2}\right) f_{y y}+\frac{11}{6}\left(u_{y y}-u_{y} u\right) f_{y}+ \\
& +\left(\frac{u_{y y y}}{2}-\frac{5\left(u_{y}\right)^{2}}{6}-\frac{5 u_{y y} u}{6}\right) f-h_{t}\left(\frac{u_{y y}}{12}+\frac{u_{y} u}{8}\right)=0 .
\end{aligned}
$$

Equation (7) allows reduction of order $u_{y}=-\frac{2}{3} u^{2}+\lambda \sqrt{u}$, where $\lambda=$ const. If $\lambda=0$ we obtain the solution in terms of elementary functions

$$
u=\frac{3}{2 y+\mu}, \quad \mu=\text { const. }
$$

From (3) we obtain

$$
g=\gamma(2 y+\mu)^{\frac{3}{2}}, \quad \gamma, \mu=\text { const. }
$$

Substituting (25) to (24), we obtain the equation of Euler type

$$
f_{\text {yyyy }}-\frac{6}{2 y+\mu} f_{y y y}+\frac{37}{(2 y+\mu)^{2}} f_{y y}-\frac{154}{(2 y+\mu)^{3}} f_{y}+\frac{324}{(2 y+\mu)^{4}} f=\frac{h_{t}}{2(2 y+\mu)^{3}} .
$$

Its general solution

$$
f=\frac{2 y+\mu}{32} h_{t}+\left(\varepsilon_{1}+\varepsilon_{2} \ln |2 y+\mu|\right)(2 y+\mu)^{3}+\left(\varepsilon_{3}+\varepsilon_{4} \ln |2 y+\mu|\right)(2 y+\mu)^{\frac{3}{2}},
$$

$\varepsilon_{i}$ are arbitrary functions of $t$. Formulas (26) and (27) give an explicit solution (but not general) of Yang-Mills equations for the metric $(1), h(t)$ is an arbitrary function. This solution gives the metric of type II.

3. For the following metric

$$
\psi=2 d t(d x+A(x, z) d t)+\left(C(z) e^{-\alpha t} d y\right)^{2}+d z^{2}, \quad \alpha=\text { const }
$$

Yang-Mills equations are very complicated, but all the same they are solved.

Components of the conformal connection matrix $\Omega$ are

$$
\begin{aligned}
& \omega^{1}=\left(\frac{1}{2}-A\right) d t-d x, \omega^{2}=\left(\frac{1}{2}+A\right) d t+d x, \omega^{3}=C e^{-\alpha t} d y, \omega^{4}=d z \\
& \omega_{1}^{2}=-A_{x}\left(\omega^{1}+\omega^{2}\right), \omega_{3}^{4}=-\frac{C_{z}}{C} \omega^{3}, \omega_{1}^{3}=\omega_{2}^{3}=-\alpha \omega^{3}, \omega_{1}^{4}=\omega_{2}^{4}=-A_{z}\left(\omega^{1}+\omega^{2}\right) .
\end{aligned}
$$

The components of the Ricci tensor and its trace are

$$
\begin{aligned}
& R_{12}=A_{z z}+\alpha^{2}-\alpha A_{x}+\frac{C_{z}}{C} A_{z}, R_{11}=R_{12}+A_{x x}, R_{22}=R_{12}-A_{x x} \\
& R_{33}=R_{44}=\frac{C_{z z}}{C}, R_{14}=R_{24}=-A_{x z}-\alpha \frac{C_{z}}{C}, R=-2 A_{x x}+2 \frac{C_{z z}}{C}
\end{aligned}
$$

The coefficients of Pfaffian forms $\omega_{i}$ :

$$
b_{12}=\frac{1}{2}\left(A_{z z}+\alpha^{2}-\alpha A_{x}+\frac{C_{z}}{C} A_{z}\right), b_{14}=b_{24}=-\frac{1}{2}\left(A_{x z}+\alpha \frac{C_{z}}{C}\right) .
$$




$$
b_{11}=\frac{1}{3} A_{x x}+\frac{C_{z z}}{6 C}+b_{12}, b_{22}=-\frac{1}{3} A_{x x}-\frac{C_{z z}}{6 C}+b_{12}, b_{33}=b_{44}=\frac{C_{z z}}{3 C}+\frac{1}{6} A_{x x},
$$

Pfaffian forms $\omega_{i}$ :

$$
\begin{aligned}
& \omega_{1}=\left(\frac{1}{3} A_{x x}+\frac{C_{z z}}{6 C}\right) \omega^{1}+\frac{1}{2}\left(A_{z z}+\alpha^{2}-\alpha A_{x}+A_{z} \frac{C_{z}}{C}\right)\left(\omega^{1}+\omega^{2}\right)-\frac{1}{2}\left(A_{x z}+\frac{\alpha C_{z}}{C}\right) \omega^{4}, \\
& \omega_{2}=-\left(\frac{1}{3} A_{x x}+\frac{C_{z z}}{6 C}\right) \omega^{2}+\frac{1}{2}\left(A_{z z}+\alpha^{2}-\alpha A_{x}+A_{z} \frac{C_{z}}{C}\right)\left(\omega^{1}+\omega^{2}\right)-\frac{1}{2}\left(A_{x z}+\frac{\alpha C_{z}}{C}\right) \omega^{4}, \\
& \omega_{3}=\left(\frac{C_{z z}}{3 C}+\frac{1}{6} A_{x x}\right) \omega^{3}, \quad \omega_{4}=-\frac{1}{2}\left(A_{x z}+\frac{\alpha C_{z}}{C}\right)\left(\omega^{1}+\omega^{2}\right)+\left(\frac{C_{z z}}{3 C}+\frac{1}{6} A_{x x}\right) \omega^{4} .
\end{aligned}
$$

Then we compute the components of the conformal curvature matrix $\Phi$. For brevity we denote $S \stackrel{\text { def }}{=} A_{x x}-\frac{C_{z z}}{C}, T \stackrel{\text { def }}{=} A_{x z}-\alpha \frac{C_{z}}{C}, L \stackrel{\text { def }}{=} A_{z z}-\alpha^{2}+\alpha A_{x}-A_{z} \frac{C_{z}}{C}$.

$$
\begin{aligned}
\Phi_{1}^{2} & =\frac{1}{3} S \omega^{1} \wedge \omega^{2}+\frac{1}{2} T\left(\omega^{1}+\omega^{2}\right) \wedge \omega^{4}, \\
\Phi_{1}^{3} & =-\frac{1}{2} L\left(\omega^{1}+\omega^{2}\right) \wedge \omega^{3}-\frac{1}{6} S \omega^{1} \wedge \omega^{3}-\frac{1}{2} T \omega^{3} \wedge \omega^{4}, \\
\Phi_{1}^{4} & =\frac{1}{2} T \omega^{1} \wedge \omega^{2}-\frac{1}{6} S \omega^{1} \wedge \omega^{4}+\frac{1}{2} L\left(\omega^{1}+\omega^{2}\right) \wedge \omega^{4}, \\
\Phi_{2}^{4} & =\frac{1}{2} L\left(\omega^{1}+\omega^{2}\right) \wedge \omega^{4}+\frac{1}{6} S \omega^{2} \wedge \omega^{4}+\frac{1}{2} T \omega^{1} \wedge \omega^{2}, \\
\Phi_{3}^{4} & =-\frac{1}{3} S \omega^{3} \wedge \omega^{4}-\frac{1}{2} T\left(\omega^{1}+\omega^{2}\right) \wedge \omega^{3}, \\
\Phi_{2}^{3} & =-\frac{1}{2} T \omega^{3} \wedge \omega^{4}+\frac{1}{6} S \omega^{2} \wedge \omega^{3}-\frac{1}{2} L\left(\omega^{1}+\omega^{2}\right) \wedge \omega^{3},
\end{aligned}
$$

$$
\begin{aligned}
\Phi_{1} & =\left(\frac{1}{3} A_{x x x}\left(A-\frac{1}{2}\right)-\left(b_{12}\right)_{x}\right) \omega^{1} \wedge \omega^{2}+\left(P-\frac{1}{4} A_{x x z}\right)\left(\omega^{1}+\omega^{2}\right) \wedge \omega^{4}+\frac{1}{6} S_{z} \omega^{1} \wedge \omega^{4}, \\
\Phi_{2} & =\left(\frac{1}{3} A_{x x x}\left(A+\frac{1}{2}\right)-\left(b_{12}\right)_{x}\right) \omega^{1} \wedge \omega^{2}+\left(P+\frac{1}{4} A_{x x z}\right)\left(\omega^{1}+\omega^{2}\right) \wedge \omega^{4}-\frac{1}{6} S_{z} \omega^{1} \wedge \omega^{3} \\
\Phi_{3} & =K\left(\omega^{1}+\omega^{2}\right) \wedge \omega^{3}-\frac{1}{6} A_{x x x} \omega^{1} \wedge \omega^{3}-\left(b_{33}\right)_{z} \omega^{3} \wedge \omega^{4}, \\
\Phi_{4} & =\frac{1}{2} A_{x x z} \omega^{1} \wedge \omega^{2}+M\left(\omega^{1}+\omega^{2}\right) \wedge \omega^{4}-\frac{1}{6} A_{x x x} \omega^{1} \wedge \omega^{4} .
\end{aligned}
$$

In the last four formulas we denoted

$$
\begin{aligned}
& P \stackrel{\text { def }}{=} \frac{1}{2} A_{z}\left(A_{x x}+\frac{C_{z z}}{C}\right)-\left(b_{12}\right)_{z}+\frac{1}{2} A_{x x z} A+A_{x} b_{14} \\
& K \stackrel{\text { def }}{=}-\frac{1}{6} A_{x x x} A+\frac{1}{2} A_{x z} \frac{C_{z}}{C}+\frac{\alpha}{2}\left(\frac{C_{z}^{2}}{C^{2}}-\frac{C_{z z}}{C}-A_{x x}\right)+\frac{1}{12} A_{x x x} \\
& M \stackrel{\text { def }}{=} \frac{1}{2}\left(A_{x z z}+\frac{\alpha C_{z z}}{C}-\frac{\alpha C_{z}^{2}}{C^{2}}-\frac{1}{3} A_{x x x} A\right)+\frac{1}{12} A_{x x x} .
\end{aligned}
$$

Petrov matrix $Q(\lambda)$ is

$$
\left(\begin{array}{ccc}
-\frac{1}{3} S+\lambda & \frac{i}{2} T & -\frac{1}{2} T \\
\frac{i}{2} T & \frac{1}{6} S+\frac{1}{2} L+\lambda & \frac{i}{2} L \\
-\frac{1}{2} T & \frac{i}{2} L & \frac{1}{6} S-\frac{1}{2} L+\lambda
\end{array}\right)
$$


It has type II when $S \neq 0$, type III when $S=0, T \neq 0$, subtype N when $S=T=0, L \neq 0$, subtype O when $S=T=L=0$.

Proceeding from the specifics of the conformal curvature matrix, it's best to start compiling Yang-Mills equations for the difference between $* \Phi_{1}$ and $* \Phi_{2}$, i.e. with the equation

$$
d\left(* \Phi_{1}-* \Phi_{2}\right)+\omega_{k} \wedge\left(* \Phi_{1}^{k}-* \Phi_{2}^{k}\right)-* \Phi_{k} \wedge\left(\omega_{1}^{k}-\omega_{2}^{k}\right)=0 .
$$

We obtain three equations

$$
\begin{gathered}
A_{x x x x}=0, \quad A_{x x x z}=0 \\
\frac{A_{x x x}}{3}\left(A_{x}+\alpha\right)-\frac{A_{x x z z}}{3}-\frac{A_{x x z} C_{z}}{3 C}-\frac{\left(A_{x x}\right)^{2}}{6}-\frac{C_{z z z z}}{6 C}+\frac{C_{z z z} C_{z}}{6 C^{2}}+\frac{1}{3}\left(\frac{C_{z z}}{C}\right)^{2}-\frac{C_{z z} C_{z}^{2}}{6 C^{3}}=0 .
\end{gathered}
$$

Equalities (29) mean that

$$
A_{x x x}=\beta=\text { const. }
$$

Taking into account these equations, Yang-Mills equations for the external form $* \Phi_{1}$ provide two new conditions

$$
\begin{gathered}
-\frac{1}{2} A_{z z z z}+A_{x x z z} A-\alpha \beta A+A_{x x z}\left(A \frac{C_{z}}{C}+\frac{5}{3} A_{z}\right)-A_{x z z} A_{x}+\alpha A_{x z z}-A_{z z z} \frac{C_{z}}{C}+ \\
+A_{x x}\left(\frac{2}{3} A_{z z}+A_{z} \frac{2 C_{z}}{3 C}+\frac{\alpha}{3} A_{x}-\frac{5}{6} \alpha^{2}\right)-A_{x z}^{2}-A_{x z} A_{x} \frac{C_{z}}{C}+\alpha A_{x z} \frac{C_{z}}{C}-\alpha A_{x} \frac{C_{z z}}{3 C}+ \\
+A_{z z}\left(\frac{C_{z}^{2}}{2 C^{2}}-\frac{2 C_{z z}}{3 C}\right)+A_{z}\left(\frac{C_{z z} C_{z}}{C^{2}}-\frac{C_{z z z}}{6 C}-\frac{C_{z}^{3}}{2 C^{3}}\right)+\alpha^{2}\left(\frac{C_{z}^{2}}{C^{2}}-\frac{2 C_{z z}}{3 C}\right)=0 \\
\frac{1}{3} A_{x x} A_{x z}+A_{x z} \frac{C_{z z}}{6 C}-\frac{2}{3} \beta A_{z}+\frac{1}{2} A_{x z z z}-\frac{\alpha}{2} A_{x x z}- \\
-A_{x z} \frac{C_{z}^{2}}{2 C^{2}}+A_{x z z} \frac{C_{z}}{2 C}+\alpha A_{x x} \frac{C_{z}}{3 C}+\alpha \frac{C_{z z z}}{2 C}-\alpha \frac{5 C_{z z} C_{z}}{6 C^{2}}=0 .
\end{gathered}
$$

Taking into account (31), Yang-Mills equations for the external form $* \Phi_{3}$ yield one new equality

$$
\frac{1}{6} A_{x x z z}-\frac{\beta}{3} A_{x}+A_{x x z} \frac{C_{z}}{2 C}-\frac{\alpha \beta}{2}+\frac{1}{3}\left(\frac{C_{z z}}{C}\right)_{z z}-\frac{1}{6}\left(\frac{C_{z z}}{C}\right)^{2}+\frac{1}{6} A_{x x}^{2}=0,
$$

and for the external form $* \Phi_{4}$ one new equation

$$
-\frac{1}{2} A_{x x z z}-A_{x x z} \frac{C_{z}}{6 C}-\frac{1}{6} A_{x x}^{2}+\frac{\beta}{3} A_{x}+\frac{C_{z z}^{2}}{6 C^{2}}-\frac{C_{z}}{3 C}\left(\frac{C_{z z}}{C}\right)_{z}+\frac{\alpha \beta}{6}=0 .
$$

Equation (31) is equivalent to

$$
A=\frac{1}{6} \beta x^{3}+f(z) x^{2}+g(z) x+h(z) .
$$

Six equations (30), (32), (33), (34), (35) and (36) connect with differential relations five functions $A, f, g, h$ and $C$. But equations (33), (34) and (35) are linearly dependent: $2 L_{1}+L_{2}=$ $L_{3}$, where $L_{1}, L_{2}, L_{3}$ are left sides of the equations (33), (34) and (35). Therefore, equation (35) may be discarded. The rest of the equations impose to functions $f, g, h$ and $C$ seven differential relations, only four of which are independent (we denote with stroke' the derivative with respect to $z)$ :

$$
f^{\prime \prime}-\frac{C^{\prime}}{C} f^{\prime}+\frac{\alpha \beta}{2}-\frac{1}{2}\left(\frac{C^{\prime \prime}}{C}\right)^{\prime \prime}+\frac{1}{2}\left(\frac{C^{\prime \prime}}{C}\right)^{\prime} \frac{C^{\prime}}{C}=0
$$




$$
\begin{gathered}
f^{\prime \prime}+\frac{1}{3} \frac{C^{\prime}}{C} f^{\prime}+\frac{2}{3} f^{2}-\frac{\beta g}{3}-\frac{\alpha \beta}{6}-\frac{1}{6}\left(\frac{C^{\prime \prime}}{C}\right)^{2}+\frac{1}{3}\left(\frac{C^{\prime \prime}}{C}\right)^{\prime} \frac{C^{\prime}}{C}=0, \\
\frac{g^{\prime \prime \prime}}{2}-\frac{2 \beta h^{\prime}}{3}-\alpha f^{\prime}+\frac{C^{\prime}}{2 C} g^{\prime \prime}+\frac{2 f g^{\prime}}{3}+\frac{2 \alpha f C^{\prime}}{3 C}+g^{\prime}\left(\frac{C^{\prime \prime}}{6 C}-\frac{1}{2}\left(\frac{C^{\prime}}{C}\right)^{2}\right)+\frac{\alpha C^{\prime \prime \prime}}{2 C}-\frac{5 \alpha C^{\prime \prime} C^{\prime}}{6 C^{2}}=0, \\
-\frac{1}{2} h^{(4)}-\frac{C^{\prime}}{C} h^{\prime \prime \prime}+h^{\prime \prime}\left(\frac{4 f}{3}-\frac{2 C^{\prime \prime}}{3 C}+\frac{1}{2}\left(\frac{C^{\prime}}{C}\right)^{2}\right)+ \\
+h^{\prime}\left(\frac{10 f^{\prime}}{3}+\frac{4 f C^{\prime}}{3 C}-\frac{C^{\prime \prime \prime}}{6 C}+\frac{C^{\prime \prime} C^{\prime}}{C^{2}}-\frac{1}{2}\left(\frac{C^{\prime}}{C}\right)^{3}\right)+h\left(2 f^{\prime \prime}+2 f^{\prime} \frac{C^{\prime}}{C}-\alpha \beta\right)-g^{\prime \prime}(g-\alpha) \\
--g^{\prime 2}-g^{\prime} g \frac{C^{\prime}}{C}+\frac{\alpha g^{\prime} C^{\prime}}{C}+g\left(\frac{2 \alpha f}{3}-\frac{\alpha C^{\prime \prime}}{3 C}\right)-\frac{5 \alpha^{2} f}{3}+\alpha^{2}\left(-\frac{2 C^{\prime \prime}}{3 C}+\left(\frac{C^{\prime}}{C}\right)^{2}\right)=0 .
\end{gathered}
$$

Without additional constraints, the system of equations (37)-(40) can not be solved explicitly. The first of these constraints is

$$
C=e^{\gamma z}, \quad \gamma=\text { const } \neq 0 .
$$

In this case system (37)-(40) is solved in terms of elementary functions, but the results are different if $\beta \neq 0$ and $\beta=0$.

At $\beta \neq 0$ we obtain solution

$$
f=\varepsilon_{1}+\frac{\alpha \beta}{2 \gamma} z, \quad g=\frac{2 f^{2}}{\beta}-\frac{\gamma^{4}}{2 \beta}, \quad h=\frac{4}{3 \beta^{2}} f^{3}-\frac{\alpha \gamma^{3}}{2 \beta} z+\varepsilon_{2} .
$$

Directly through $x$ and $z$ formula (36) can be written as

$$
A=\frac{\beta}{6}\left(x+\frac{\alpha}{\gamma} z\right)^{3}+\varepsilon_{1}\left(x+\frac{\alpha}{\gamma} z\right)^{2}+\frac{2}{\beta}\left(\left(\varepsilon_{1}\right)^{2}-\frac{\gamma^{4}}{4}\right)\left(x+\frac{\alpha}{\gamma} z\right)+\frac{4\left(\varepsilon_{1}\right)^{3}}{\beta^{2}}+\varepsilon_{2},
$$

$\varepsilon_{1}, \varepsilon_{2}, \beta, \gamma$ are arbitrary constants, but $\beta \neq 0$ and $\gamma \neq 0$. Formulas (42) and (41) together with (28) give Yang-Mills metric of type II.

From (41) and $\beta=0$ it follows, that (by virtue of equations (37) and (38)) there are only two possibilities for the function $f(z)$ :

$$
f= \pm \frac{1}{2} \gamma^{2}
$$

If the sign is + the following solution to the equations (39) and (40) is obtained

$$
\begin{gathered}
g=\varepsilon_{1}+\varepsilon_{2} z+\varepsilon_{3} e^{-\gamma z} \\
h=\varepsilon_{4}+\varepsilon_{5} e^{-\gamma z}+\varepsilon_{6} e^{\gamma z}+\varepsilon_{7} e^{-2 \gamma z}+\frac{\left(\varepsilon_{3}\right)^{2}}{3 \gamma} z e^{-2 \gamma z}+ \\
+\frac{\varepsilon_{2} \varepsilon_{3}}{\gamma^{2}} z e^{-\gamma z}+z\left(\frac{\left(\varepsilon_{2}\right)^{2}}{2 \gamma^{2}} z-\frac{\alpha \varepsilon_{2}}{\gamma^{2}}+\frac{\varepsilon_{1} \varepsilon_{2}}{\gamma^{2}}+\frac{\alpha^{2}}{2 \gamma}+\frac{\left(\varepsilon_{2}\right)^{2}}{2 \gamma^{3}}\right) .
\end{gathered}
$$

Here $\varepsilon_{1}, \ldots, \varepsilon_{7}, \gamma$ are arbitrary constants, but $\gamma \neq 0$. Formulas (44), (43) (with + in the right side), (41), (36) and (28) give Yang-Mills metric of type III.

Now consider the case with a minus in the right side of (43). Then the solution of (39) and 
(40) is

$$
\begin{gathered}
g=\varepsilon_{1}+\varepsilon_{2} e^{\lambda_{1} \gamma z}+\varepsilon_{3} e^{\lambda_{2} \gamma z}-\alpha \gamma z \\
h=e^{-\frac{1}{2} \gamma z}\left(\varepsilon_{6} \cos \sqrt{\frac{5}{12}} \gamma z+\varepsilon_{7} \sin \sqrt{\frac{5}{12}} \gamma z\right)+ \\
+\frac{\left(\varepsilon_{2}\right)^{2}}{2 \gamma^{2}\left(\lambda_{1}-3\right)} e^{2 \lambda_{1} \gamma z}+\frac{\left(\varepsilon_{3}\right)^{2}}{2 \gamma^{2}\left(\lambda_{2}-3\right)} e^{2 \lambda_{2} \gamma z}+\varepsilon_{4}+\varepsilon_{5} e^{-\gamma z}+ \\
+\frac{\varepsilon_{2}}{\gamma^{2}}\left(\alpha z \gamma-\alpha \lambda_{1}-\varepsilon_{1}\right) e^{\lambda_{1} \gamma z}+\frac{\varepsilon_{3}}{\gamma^{2}}\left(\alpha z \gamma-\alpha \lambda_{2}-\varepsilon_{1}\right) e^{\lambda_{2} \gamma z}+\left(\frac{\alpha \varepsilon_{1}}{\gamma}-\frac{1}{2} \alpha^{2} z\right) z .
\end{gathered}
$$

Here $\varepsilon_{1}, \ldots, \varepsilon_{7}, \gamma$ are arbitrary constants, but $\gamma \neq 0 ; \lambda_{1}=-\frac{1}{2}+\sqrt{\frac{19}{12}}, \lambda_{2}=-\frac{1}{2}-\sqrt{\frac{19}{12}}$. Formulas (44), (43) (with minus in the right side), (41), (36) and (28) give Yang-Mills metric of type II.

Consider now instead of (41) other constraints: $C^{\prime \prime}=0, S=0$. Then we obtain the following solution

$$
\begin{gathered}
C=\varepsilon_{1} z+\varepsilon_{2}, \quad f=0, \quad \beta=0, \quad g=\varepsilon_{3}\left(z+\frac{\varepsilon_{2}}{\varepsilon_{1}}\right)^{2}+\varepsilon_{4} \ln \left(z+\frac{\varepsilon_{2}}{\varepsilon_{1}}\right)+\varepsilon_{5}, \\
h=\varepsilon_{9} \ln \left(z+\frac{\varepsilon_{2}}{\varepsilon_{1}}\right)-\frac{\left(\varepsilon_{3}\right)^{2}}{36}\left(z+\frac{\varepsilon_{2}}{\varepsilon_{1}}\right)^{6}+\frac{\varepsilon_{3}}{16}\left(z+\frac{\varepsilon_{2}}{\varepsilon_{1}}\right)^{4}\left(2 \alpha+\varepsilon_{4}-2 \varepsilon_{5}-2 \varepsilon_{4} \ln \left(z+\frac{\varepsilon_{2}}{\varepsilon_{1}}\right)\right)+ \\
+\varepsilon_{8}+\frac{1}{8}\left(z+\frac{\varepsilon_{2}}{\varepsilon_{1}}\right)^{2}\left(4 \varepsilon_{6}-2 \varepsilon_{7}+\alpha^{2}-\left(\varepsilon_{4}\right)^{2}+\left(4 \varepsilon_{7}-2 \alpha^{2}+2\left(\varepsilon_{4}\right)^{2}\right) \ln \left(z+\frac{\varepsilon_{2}}{\varepsilon_{1}}\right)\right) .
\end{gathered}
$$

Here $\varepsilon_{1}, \ldots, \varepsilon_{9}$ are arbitrary constants, but $\varepsilon_{1} \neq 0$. We have received Yang-Mills metric of type III.

Thus, the metric (28) has provided us with two series of Yang-Mills metric of types II and III.

Another remarkable feature of the metric (28) is that at suitable function $A(x, z)$ and $C(z)$ it gives Einstein metric of type II, which is a rare phenomenon. We do not give the full solution, but only one particular case:

$$
A=-\frac{1}{2} \gamma^{2} x^{2}+x\left(\varepsilon_{1}-\alpha \gamma z\right)+\varepsilon_{4}+\varepsilon_{5} e^{-\gamma z}+z\left(\frac{\alpha \varepsilon_{1}}{\gamma}-\frac{1}{2} \alpha^{2} z\right), \quad C=e^{\gamma z} .
$$

This gives Einstein metric of type II, which is a particular case of solution (45) at $\varepsilon_{2}=\varepsilon_{3}=$ $\varepsilon_{6}=\varepsilon_{7}=0$.

\section{Metric of type III, satisfying Yang-Mills equations}

Let's investigate the metric

$$
\psi=2 d t(d x+B(t, z) d y+A(t, z) d t)+(C(t) d y)^{2}+d z^{2},
$$

which, as we shall see, may be of types III, $\mathrm{N}$ or $\mathrm{O}$. The components of conformal connection matrix $\Omega$ are

$$
\begin{aligned}
\omega^{1} & =\left(\frac{1}{2}-A\right) d t-d x-B d y, \omega^{2}=\left(\frac{1}{2}+A\right) d t+d x+B d y, \omega^{3}=C d y, \omega^{4}=d z, \\
\omega_{1}^{3} & =\omega_{2}^{3}=\frac{\dot{B}}{C}\left(\omega^{1}+\omega^{2}\right)+\frac{\dot{C}}{C} \omega^{3}+\frac{B^{\prime}}{2 C} \omega^{4}, \omega_{1}^{2}=0, \omega_{1}^{4}=\omega_{2}^{4}=-A^{\prime}\left(\omega^{1}+\omega^{2}\right)-\frac{B^{\prime}}{2 C} \omega^{3}, \\
\omega_{3}^{4} & =-\frac{B^{\prime}}{2 C}\left(\omega^{1}+\omega^{2}\right), \omega_{1}=\omega_{2}=K\left(\omega^{1}+\omega^{2}\right)+\frac{B^{\prime \prime}}{4 C} \omega^{3}, \omega_{3}=\frac{B^{\prime \prime}}{4 C}\left(\omega^{1}+\omega^{2}\right), \omega_{4}=0,
\end{aligned}
$$


where dot denotes the derivative with respect to $t$, stroke ' denotes derivative with respect to $z$, $K \stackrel{\text { def }}{=} \frac{1}{2}\left(A^{\prime \prime}+\frac{\ddot{C}}{C}-\frac{1}{2}\left(\frac{B^{\prime}}{C}\right)^{2}\right)$.

The components of the conformal curvature matrix $\Phi$ are:

$$
\begin{aligned}
& \Phi_{1}^{2}=\frac{B^{\prime \prime}}{4 C}\left(\omega^{1}+\omega^{2}\right) \wedge \omega^{3}, \quad \Phi_{3}^{4}=\frac{B^{\prime \prime}}{4 C}\left(\omega^{1}+\omega^{2}\right) \wedge \omega^{4} \\
& \Phi_{1}^{3}=\Phi_{2}^{3}=\frac{B^{\prime \prime}}{4 C} \omega^{1} \wedge \omega^{2}+S\left(\omega^{1}+\omega^{2}\right) \wedge \omega^{3}-T\left(\omega^{1}+\omega^{2}\right) \wedge \omega^{4} \\
& \Phi_{1}^{4}=\Phi_{2}^{4}=\frac{B^{\prime \prime}}{4 C} \omega^{3} \wedge \omega^{4}-S\left(\omega^{1}+\omega^{2}\right) \wedge \omega^{4}-T\left(\omega^{1}+\omega^{2}\right) \wedge \omega^{3}
\end{aligned}
$$

where $S \stackrel{\text { def }}{=} \frac{1}{2}\left(\frac{\ddot{C}}{C}-A^{\prime \prime}\right), T \stackrel{\text { def }}{=} \frac{1}{2}\left(\frac{\dot{B}^{\prime}}{C}+\frac{B^{\prime} \dot{C}}{C^{2}}\right)$,

$$
\begin{aligned}
\Phi_{1}= & \Phi_{2}=\frac{1}{4}\left(\left(\frac{B^{\prime \prime}}{C}\right)+\frac{2 B^{\prime \prime} \dot{C}}{C^{2}}\right)\left(\omega^{1}+\omega^{2}\right) \wedge \omega^{3}+ \\
& +\left(\frac{B^{\prime \prime} B^{\prime}}{8 C^{2}}-K^{\prime}\right)\left(\omega^{1}+\omega^{2}\right) \wedge \omega^{4}-\frac{B^{\prime \prime \prime}}{4 C} \omega^{3} \wedge \omega^{4}, \\
\Phi_{3}= & -\frac{B^{\prime \prime \prime}}{4 C}\left(\omega^{1}+\omega^{2}\right) \wedge \omega^{4}, \quad \Phi_{4}=0 .
\end{aligned}
$$

Petrov matrix $Q(\lambda)$ is

$$
\left(\begin{array}{ccc}
\lambda & -\frac{B^{\prime \prime}}{4 C} & -\frac{i B^{\prime \prime}}{4 C} \\
-\frac{B^{\prime \prime}}{4 C} & -S-i T+\lambda & T-i S \\
-\frac{i B^{\prime \prime}}{4 C} & T-i S & S+i T+\lambda
\end{array}\right)
$$

This is a matrix of type III if $B^{\prime \prime} \neq 0$; of type $\mathrm{N}$ if $B^{\prime \prime}=0$ and of type $\mathrm{O}$ if $B^{\prime \prime}=0, A^{\prime \prime}=\frac{\ddot{C}}{C}$, $B^{\prime} C=\varepsilon(z)$, where $\varepsilon(z)$ is arbitrary function of $z$.

Yang-Mills equations for the external form $* \Phi_{4}$ are satisfied identically, for the form $* \Phi_{3}$ they are reduced to a single equation $\frac{\partial^{4} B}{\partial z^{4}}=0$. External forms $* \Phi_{1}$ and $* \Phi_{2}$ also yield only one equation $\frac{\partial^{4} A}{\partial z^{4}}=\frac{3}{2}\left(\frac{B^{\prime \prime}}{C}\right)^{2}+\frac{3 B^{\prime \prime \prime} B^{\prime}}{2 C^{2}}$. The system of these two equations has a solution

$$
\begin{aligned}
& B=\beta_{0} z^{3}+\beta_{1} z^{2}+\beta_{2} z+\beta_{3}, \\
& A=\frac{1}{C^{2}}\left[\frac{9\left(\beta_{0}\right)^{2}}{40} z^{6}+\frac{9 \beta_{0} \beta_{1}}{20} z^{5}+\left(\frac{3 \beta_{0} \beta_{2}}{8}+\frac{\left(\beta_{1}\right)^{2}}{4}\right) z^{4}\right]+\alpha_{0} z^{3}+\alpha_{1} z^{2}+\alpha_{2} z+\alpha_{3},
\end{aligned}
$$

where $\beta_{0}, \beta_{1}, \beta_{2}, \beta_{3}, \alpha_{0}, \alpha_{1}, \alpha_{2}, \alpha_{3}, C$ are arbitrary functions of $t$.

Mertic (46) is Einstein metric when $B^{\prime \prime}=0, A^{\prime \prime}+\frac{\ddot{C}}{C}-\frac{1}{2}\left(\frac{B^{\prime}}{C}\right)^{2}=0$.

\section{References}

[1] A.Z.Petrov, New methods in General Relativity, 1966, Mosscow, Nauka (in Russian). 
[2] Yu.S.Vladimirov, Geometrophysics, BINOM, Moscow, 2010 (in Russian).

[3] L.N.Krivonosov, V.A.Luk'yanov, The full solution of Yang-Mills equations for the centralsymmetric metrics, Journal of Siberian Federal University. Mathematics \& Physics, 4(2011), no. 3, 350-362 (in Russian).

[4] L.N.Krivonosov, V.A.Luk'yanov, Purely time-dependent solutions to the Yang-Mills equations on a 4-dimensional manifold with conformal torsion-free connection, Journal of Siberian Federal University. Mathematics \& Physics, 6(2013), no. 1, 40-52.

[5] M.Korzyjnski, J.Levandowski, The Normal Conformal Cartan Connection and the Bach Tensor, arXiv:gr-qc/0301096v3, 2003.

\section{Решение уравнений Янга-Миллса для 4-метрик типов II, N, III Петрова}

\section{Леонид Н. Кривоносов \\ Вячеслав А.Лукьянов}

$\overline{\text { Приводятся по 4 серии 4-метрик для каждого из видов II, N, III, удовлетворяющих уравнениям }}$ Янга-Миллса.

Ключевые слова: уравнения Эйнштейна, уравнения Янга-Миллса, многообразие конформной связности с кручением и без кручения. 